\title{
Morphological instability during steady electrodeposition at overlimiting currents
}

\author{
Nielsen, Christoffer Peder; Bruus, Henrik
}

Published in:

Physical Review E

Publication date:

2015

Document Version

Publisher's PDF, also known as Version of record

Link back to DTU Orbit

Citation (APA):

Nielsen, C. P., \& Bruus, H. (2015). Morphological instability during steady electrodeposition at overlimiting currents. Physical Review E, 92(5), [052310].

\section{General rights}

Copyright and moral rights for the publications made accessible in the public portal are retained by the authors and/or other copyright owners and it is a condition of accessing publications that users recognise and abide by the legal requirements associated with these rights.

- Users may download and print one copy of any publication from the public portal for the purpose of private study or research.

- You may not further distribute the material or use it for any profit-making activity or commercial gain

- You may freely distribute the URL identifying the publication in the public portal

If you believe that this document breaches copyright please contact us providing details, and we will remove access to the work immediately and investigate your claim. 


\title{
Morphological instability during steady electrodeposition at overlimiting currents
}

\author{
Christoffer P. Nielsen* and Henrik Bruus ${ }^{\dagger}$ \\ Department of Physics, Technical University of Denmark, DTU Physics Building 309, DK-2800 Kongens Lyngby, Denmark
}

(Received 27 May 2015; revised manuscript received 25 August 2015; published 16 November 2015)

\begin{abstract}
We present a linear stability analysis of a planar metal electrode during steady electrodeposition. We extend the previous work of Sundstrom and Bark by accounting for the extended space-charge density, which develops at the cathode once the applied voltage exceeds a few thermal voltages. In accordance with Chazalviel's conjecture, the extended space-charge region is found to greatly affect the morphological stability of the electrode. To supplement the numerical solution of the stability problem, we have derived analytical expressions valid in the limit of low and high voltage, respectively.
\end{abstract}

DOI: 10.1103/PhysRevE.92.052310

PACS number(s): 82.45.Qr, 82.45.Gj, 61.43.Hv

\section{INTRODUCTION}

One of the most interesting aspects of systems, involving transport between matter in different phases, is their tendency to become morphologically unstable and develop ramified growth patterns. Well-known examples include snowflake formation and dendritic growth during metal solidification $[1,2]$. A particularly interesting and challenging growth problem is encountered in electrodeposition from an electrolyte onto an electrode [3-11]. Whereas snowflake formation and solidification are mainly driven by diffusion of water vapor and heat, respectively [1,2], electrodeposition is driven by electromigration in addition to diffusion $[12,13]$. For this reason, the electrodeposition rate can be driven to exceed the diffusion limit, at which point the system enters a nonlinear regime not encountered in the purely diffusion-driven systems. One of the features of this nonlinear regime is the development of a nonequilibrium space-charge region, which extends from the cathode into the electrolyte [12,14-16]. This extended space-charge region significantly affects the transport in the system, and it is a central component in the well-known electroosmotic instability [17-19]. In 1990 Chazalviel realized that the extended space-charge region is crucial to the understanding of ramified growth during electrodeposition [12]. Nevertheless, there has been very little work which actually takes this effect into account.

In this paper we investigate the morphological stability of the cathode during electrod position in both the linear and the nonlinear regime. We follow the approach of Sundstrom and Bark [20] and investigate steady electrodeposition in a system composed of an electrolyte sandwiched between two, initially planar, metal electrodes. We solve the stability problem numerically and find that the higher the applied voltage difference is, the more unstable the electrode surface becomes. Also, the most unstable wavelength becomes smaller as the voltage bias is increased. In the numerical solution we employ the widely used Butler-Volmer reaction expression with constant charge-transfer coefficients. Apart from the extensive use of this model in the literature, the main reason for choosing it here is its conceptual simplicity and reliance on only a few reaction parameters, allowing for a basic understanding of the system.

\footnotetext{
*chnie@fysik.dtu.dk

†bruus@fysik.dtu.dk
}

Also, while the model may not describe reaction kinetics as well as more elaborate models, it nevertheless correctly captures the exponential dependence on overpotential, which is an almost universal feature in reaction models $[21,22]$.

In addition to solving the stability problem numerically, we derive analytical expressions for the perturbation growth rate, valid in the low and high voltage limit, respectively. In deriving these expressions, we make use of an accurate analytical model for the extended space-charge region, which we presented in a recent paper [16]. While our numerical results are restricted to the standard Butler-Volmer model, our analytical models cover a more general class of reaction models, including, e.g., Marcus kinetics and Butler-Volmer-Frumkin kinetics.

\section{MODEL SYSTEM}

Following Sundstrom and Bark [20], we consider a binary electrolyte trapped between two coplanar metal electrodes at $x=0$ and $x=2 L$. The electrolyte has initial concentration $c_{0}$ and is assumed symmetric with valence $Z$. The coordinate system is moving in the negative $x$ direction with velocity $U$, which is the rate of deposited layer growth and thus related to the average deposition rate on the electrodes. We consider the dilute solution limit, in which the effect of the moving coordinate system is negligible everywhere except in the surface evolution equation. A sketch of the system is shown in Fig. 1.

In the analysis, we investigate the stability of the electrodes under $y$-dependent perturbations along the $x$ direction. However, our analysis is general and applies to perturbations along any direction in the $y z$ plane.

\section{GOVERNING EQUATIONS}

The current densities of either ion are given as

$$
\begin{aligned}
2 \boldsymbol{J}_{ \pm} & =-c_{ \pm} \nabla \mu_{ \pm}, \\
\mu_{ \pm} & =\ln \left(c_{ \pm}\right) \pm Z \phi,
\end{aligned}
$$

where we have nondimensionalized the currents $\boldsymbol{J}_{ \pm}$by the limiting currents $2 D_{ \pm} c_{0} / L$, the electrochemical potentials $\mu_{ \pm}$by $k_{\mathrm{B}} T$, the electric potential $\phi$ by the thermal voltage $V_{\mathrm{T}}=k_{\mathrm{B}} T / e$, the coordinates by half the electrode spacing $L$, and the concentrations $c_{ \pm}$by the initial concentration $c_{0}$. Normalizing the time by the diffusion time $t_{0}=L^{2} /\left(2 D_{+}\right)$, 


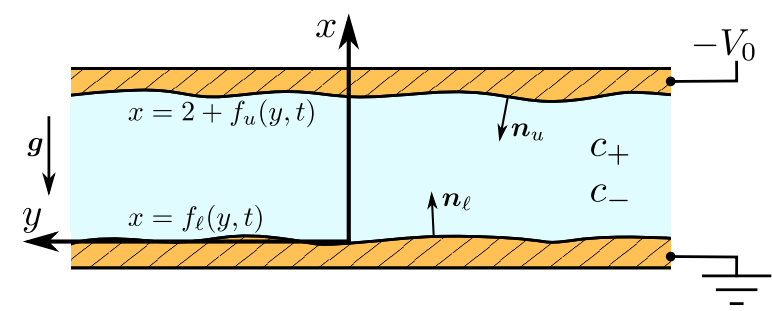

FIG. 1. (Color online) Sketch of the studied system with lower and upper electrode surfaces at $x=f_{\ell}(y, t)$ and $x=2+f_{u}(y, t)$, respectively. The coordinates are given relative to the moving frame of reference, following the mean rate of deposition on the electrode surfaces. The coordinates are also normalized by half the electrode spacing $L$.

the nondimensionalized ion-conservation equations become

$$
\frac{D_{+}}{D_{ \pm}} \partial_{t} c_{ \pm}=-\nabla \cdot J_{ \pm} \cdot
$$

At the electrodes, the current of anions vanishes, while the current of cations is given by a reaction expression

$$
\begin{aligned}
& \boldsymbol{n}_{p} \cdot \boldsymbol{J}_{-}=0, \\
& \boldsymbol{n}_{p} \cdot \boldsymbol{J}_{+}=-R_{p},
\end{aligned}
$$

where $R_{p}$ is the reaction rate at the lower and upper electrode, respectively, as indicated by the subscript $p=\ell, u$. We model the reaction rates $R_{u}$ and $R_{\ell}$ using a reaction expression of the general form

$$
R_{p}=K_{0}\left[c_{+} e^{-\bar{\gamma} \kappa+\alpha_{\mathrm{c}}\left(\phi, c_{+}\right) Z\left(\phi+V_{p}\right)}-e^{-\bar{\gamma} \kappa-\alpha_{\mathrm{a}}\left(\phi, c_{+}\right) Z\left(\phi+V_{p}\right)}\right] .
$$

Here $K_{0}$ is the dimensionless version of the dimensionfull rate constant $k_{0}$ for the electrode reaction,

$$
K_{0}=\frac{k_{0}}{2 D_{+} c_{0} / L},
$$

$V_{p}$ is the normalized electrode potential, $\kappa$ is the normalized curvature of the surface, and $\bar{\gamma}$ is the nondimensionalized version of the dimensionfull surface energy $\gamma$,

$$
\bar{\gamma}=\frac{a^{3} \gamma}{k_{\mathrm{B}} T L} .
$$

We allow the cathodic and anodic charge-transfer coefficients $\alpha_{\mathrm{c}}$ and $\alpha_{\mathrm{a}}$ to vary with the potential $\phi$ and the cation concentration $c_{+}$. In this way, Eq. (4) represents a wide range of reaction models from classical Butler-Volmer kinetics to Marcus kinetics and Butler-Volmer-Frumkin kinetics [20-22]. In agreement with most realistic reaction models, we do impose one restriction on the charge transfer coefficients, namely, that they vary slowly as a function of $\phi$ and $\ln \left(c_{+}\right)$,

$$
\begin{aligned}
\partial_{\phi}\left\{\alpha_{\mathrm{c}}\left(\phi, c_{+}\right) \phi\right\} & \approx \alpha_{\mathrm{c}}\left(\phi, c_{+}\right), \\
\partial_{\ln \left(c_{+}\right)}\left\{\alpha_{\mathrm{c}}\left(\phi, c_{+}\right) \ln \left(c_{+}\right)\right\} & \approx \alpha_{\mathrm{c}}\left(\phi, c_{+}\right) .
\end{aligned}
$$

The electrostatic part of the problem is governed by the Poisson equation,

$$
2 \bar{\lambda}_{\mathrm{D}}^{2} \nabla^{2} \phi=-\rho=-Z c_{+}+Z c_{-}
$$

where the nondimensional Debye length $\bar{\lambda}_{\mathrm{D}}$ is given as

$$
\bar{\lambda}_{\mathrm{D}}=\frac{\lambda_{\mathrm{D}}}{L}, \quad \text { with } \quad \lambda_{\mathrm{D}}=\sqrt{\frac{k_{\mathrm{B}} T \epsilon_{\mathrm{w}}}{2 e^{2} c_{0}}} .
$$

For simplicity, and to be in accordance with most previous work, we choose not to explicitly model the Debye layers adjoining the electrodes. Instead, we apply the boundary conditions (3) just outside the Debye layer. Following Ref. [23] we implement the boundary condition

$$
\boldsymbol{n}_{u} \cdot \nabla c_{+}=0
$$

at the upper electrode, to indicate the minimum in $c_{+}$at the outer edge of the Debye layer. We note that the Debye layers can be included implicitly by including the Frumkin correction to the reaction model. This correction can be implemented by an appropriate choice of the charge-transfer coefficients [22]. Together with Eq. (3b), condition (11) corresponds to ascribing the entire current into the upper electrode to electromigration.

Finally, since the anions cannot enter or leave the system the total number of anions is conserved,

$$
\int_{\Omega}\left(c_{-}-1\right) \mathrm{d} V=0 .
$$

We introduce functions $x=f_{p}(y)$ describing the position of the upper and lower electrode $u$ and $\ell$. The time evolution of $f_{p}$ is determined by the single-ion volume $a^{3}$ and the current into the electrode,

$$
\begin{aligned}
\left(\partial_{t} f_{\ell}-U\right) \boldsymbol{e}_{x} \cdot \boldsymbol{n}_{\ell} & =-a^{3} c_{0} \boldsymbol{n}_{\ell} \cdot \boldsymbol{J}_{+}, \quad \text { anode } \\
\left(\partial_{t} f_{u}-U\right) \boldsymbol{e}_{x} \cdot \boldsymbol{n}_{u}=-a^{3} c_{0} \boldsymbol{n}_{u} \cdot \boldsymbol{J}_{+}, & \text {cathode. }
\end{aligned}
$$

Here the filling factor $a^{3} c_{0}$ is much less than unity, since we are dealing with dilute solutions. The normalized velocity $U$ of the coordinate system accounts for the mean current into or out of the electrodes, and $\partial_{t} f_{p}$ accounts for local deviations from the mean current.

The curvature $\kappa$ and the normal vectors are related to the surface function $f_{p}$ by

$$
\begin{gathered}
\boldsymbol{n}_{\ell}=\frac{\boldsymbol{e}_{x}-\boldsymbol{e}_{y} \partial_{y} f_{\ell}}{\sqrt{1+\left(\partial_{y} f_{\ell}\right)^{2}}}, \quad \boldsymbol{n}_{u}=\frac{-\boldsymbol{e}_{x}+\boldsymbol{e}_{y} \partial_{y} f_{u}}{\sqrt{1+\left(\partial_{y} f_{u}\right)^{2}}}, \\
\kappa_{\ell}=\frac{\partial_{y}^{2} f_{\ell}}{\sqrt{1+\left(\partial_{y} f_{\ell}\right)^{2}}}, \quad \kappa_{u}=-\frac{\partial_{y}^{2} f_{u}}{\sqrt{1+\left(\partial_{y} f_{u}\right)^{2}}} .
\end{gathered}
$$

In defining the above equations and boundary conditions, we have chosen slightly different normalizations than in Ref. [20], the main difference being that we allow for a nonzero space charge density.

\section{PERTURBATION}

The stability of the electrodes is investigated using linear perturbation theory; that is, we impose a small perturbation on a steady-state base state and investigate how the perturbation evolves. The base state is identified by a superscript " 0 " and 
the first-order perturbation by superscript "1",

$$
\begin{aligned}
f_{p}(y, t) & \approx f_{p}^{1}(y, t), \\
c_{ \pm}(x, y, t) & \approx c_{ \pm}^{0}(x)+c_{ \pm}^{1}(x, y, t), \\
\phi(x, y, t) & \approx \phi^{0}(x)+\phi^{1}(x, y, t) .
\end{aligned}
$$

In first-order perturbation theory, we substitute the secondorder factor $\sqrt{1+\left(\partial_{y} f_{p}\right)^{2}}$ in Eq. (14) by unity,

$$
\begin{array}{ll}
\boldsymbol{n}_{\ell} \approx \boldsymbol{e}_{x}-\boldsymbol{e}_{y} \partial_{y} f_{\ell}^{1}, & \boldsymbol{n}_{u} \approx-\boldsymbol{e}_{x}+\boldsymbol{e}_{y} \partial_{y} f_{u}^{1}, \\
\kappa_{\ell} \approx \partial_{y}^{2} f_{\ell}^{1}, & \kappa_{u} \approx-\partial_{y}^{2} f_{u}^{1} .
\end{array}
$$

To find the field values at the perturbed surface, we Taylor expand to first order and obtain

$$
\phi\left(f_{\ell}^{1}, y, t\right) \approx \phi^{0}(0)+\left.\partial_{x} \phi\right|_{0} f_{\ell}^{1}(y, t)+\phi^{1}(0, y, t),
$$

$\left.\nabla \phi\left(f_{\ell}^{1}, y, t\right) \approx \partial_{y} \phi^{1}\right|_{0} \boldsymbol{e}_{y}+\left(\left.\partial_{x} \phi^{0}\right|_{0}+\left.\partial_{x}^{2} \phi^{0}\right|_{0} f_{\ell}^{1}+\left.\partial_{x} \phi^{1}\right|_{0}\right) \boldsymbol{e}_{x}$.

Similar expressions apply for $c_{ \pm}$and at the upper electrode. Evaluating the reaction rate at the lower electrode and expanding to first order, we find

$$
\begin{aligned}
R_{\ell} \approx & R_{\ell}^{0}+R_{\ell}^{1}, \\
\frac{R_{\ell}^{0}}{K_{0}}= & c_{+}^{0} e^{\alpha_{\mathrm{c}} Z\left(\phi^{0}+V_{\ell}\right)}-e^{-\alpha_{\mathrm{a}} Z\left(\phi^{0}+V_{\ell}\right)}, \\
\frac{R_{\ell}^{1}}{K_{0}}= & e^{\alpha_{\mathrm{c}} Z\left(\phi^{0}+V_{\ell}\right)}\left\{c_{+}^{1}+\partial_{x} c_{+}^{0} f_{\ell}^{1}\right. \\
& \left.+c_{+}^{0}\left(\alpha_{\mathrm{a}}+\alpha_{\mathrm{c}}\right) Z\left[\phi^{1}+\partial_{x} \phi^{0} f_{\ell}^{1}\right]\right\} \\
& +\frac{R_{\ell}^{0}}{K_{0}}\left\{-\bar{\gamma} \partial_{y}^{2} f_{\ell}^{1}-\alpha_{\mathrm{a}} Z\left[\phi^{1}+\partial_{x} \phi^{0} f_{\ell}^{1}\right]\right\},
\end{aligned}
$$

where all fields are evaluated at $x=0$, and the expression for $R_{\ell}^{0}$ was used to simplify the expression for $R_{\ell}^{1}$. Since the charge-transfer coefficients vary slowly with $\phi$ and $\ln \left(c_{+}\right)$, we have neglected their first order contributions. Similar expressions apply at the upper electrode.

Hence, the full zeroth-order problem becomes

$$
\begin{aligned}
0 & =-\partial_{x} J_{ \pm}^{0}, \\
2 J_{ \pm}^{0} & =-\partial_{x} c_{ \pm}^{0} \mp Z c_{ \pm}^{0} \partial_{x} \phi^{0}, \\
2 \bar{\lambda}_{\mathrm{D}}^{2} \partial_{x}^{2} \phi^{0} & =-Z\left(c_{+}^{0}-c_{-}^{0}\right)=-\rho^{0},
\end{aligned}
$$

with the following boundary conditions and constraints:

$$
\begin{array}{rlrl}
J_{-}^{0}(0) & =0, & J_{-}^{0}(2) & =0, \\
J_{+}^{0}(0) & =-R_{\ell}^{0}, \quad J_{+}^{0}(2)=R_{u}^{0}, \\
\int_{0}^{2}\left(c_{-}^{0}-1\right) d x & =0, \quad \partial_{x} c_{+}^{0}(2)=0,
\end{array}
$$

and the mean growth velocity $U$ derived from Eq. (13),

$$
U=a^{3} c_{0} J_{+}^{0}
$$

Similarly, the first-order problem is given by

$$
\begin{aligned}
\frac{D_{+}}{D_{ \pm}} \partial_{t} c_{ \pm}^{1} & =-\nabla \cdot J_{ \pm}^{1}, \\
2 J_{ \pm}^{1} & =-\nabla c_{ \pm}^{1} \mp Z c_{ \pm}^{0} \nabla \phi^{1} \mp Z c_{ \pm}^{1} \nabla \phi^{0} \\
2 \bar{\lambda}_{\mathrm{D}}^{2} \nabla^{2} \phi^{1} & =-Z\left(c_{+}^{1}-Z c_{-}^{1}\right),
\end{aligned}
$$

and the boundary conditions,

$$
\begin{array}{rlrl}
\boldsymbol{e}_{x} \cdot \boldsymbol{J}_{-}^{1}(2) & =0, & \boldsymbol{e}_{x} \cdot \boldsymbol{J}_{-}^{1}(0)=0, \\
\boldsymbol{e}_{x} \cdot \boldsymbol{J}_{+}^{1}(2) & =R_{u}^{1}, & \boldsymbol{e}_{x} \cdot \boldsymbol{J}_{+}^{1}(0)=-R_{\ell}^{1}, \\
\partial_{x}^{2} c_{+}^{0}(2) f_{u}^{1}+\partial_{x} c_{+}^{1}(2) & =0, & &
\end{array}
$$

together with the first-order electrode growth rates $\partial_{t} f_{\ell}^{1}$ and $\partial_{t} f_{u}^{1}$ derived from Eq. (13),

$$
\partial_{t} f_{\ell}^{1}=a^{3} c_{0} R_{\ell}^{1}, \quad \partial_{t} f_{u}^{1}=-a^{3} c_{0} R_{u}^{1} .
$$

To find the eigenmodes, we make the following harmonic ansatz for the first-order fields:

$$
\begin{aligned}
c_{ \pm}^{1}(x, y, t) & =c_{ \pm}^{*}(x) e^{\Gamma t+i k y}, \\
\phi^{1}(x, y, t) & =\phi^{*}(x) e^{\Gamma t+i k y}, \\
f_{p}^{1}(y, t) & =F_{p} e^{\Gamma t+i k y},
\end{aligned}
$$

where $\Gamma$ is the nondimensional growth rate of the perturbation, and $k$ is the wave number of the transverse eigenmode. For convenience we also define

$$
R_{p}^{1}=R_{p}^{*} e^{\Gamma t+i k y} .
$$

With this ansatz, the first-order bulk equations become

$$
\begin{aligned}
2 \frac{D_{+}}{D_{ \pm}} \Gamma c_{ \pm}^{*}= & -k^{2}\left(c_{ \pm}^{*} \pm Z c_{ \pm}^{0} \phi^{*}\right) \\
& +\partial_{x}\left\{\partial_{x} c_{ \pm}^{*} \pm Z c_{ \pm}^{*} \partial_{x} \phi^{0} \pm Z c_{ \pm}^{0} \partial_{x} \phi^{*}\right\}
\end{aligned}
$$

$$
2 \bar{\lambda}_{\mathrm{D}}^{2}\left(\partial_{x}^{2} \phi^{*}-k^{2} \phi^{*}\right)=-Z\left(c_{+}^{*}-c_{-}^{*}\right),
$$

and the first-order reaction rate at the lower electrode is

$$
\begin{aligned}
\frac{R_{\ell}^{*}}{K_{0}}= & e^{\alpha_{\mathrm{c}} Z\left(\phi^{0}+V_{\ell}\right)}\left\{c_{+}^{*}+\partial_{x} c_{+}^{0} F_{\ell}\right. \\
& \left.+c_{+}^{0}\left(\alpha_{\mathrm{a}}+\alpha_{\mathrm{c}}\right) Z\left[\phi^{*}+\partial_{x} \phi^{0} F_{\ell}\right]\right\} \\
& +\frac{R_{\ell}^{0}}{K_{0}}\left\{-\bar{\gamma} k^{2} F_{\ell}-\alpha_{\mathrm{a}} Z\left[\phi^{*}+\partial_{x} \phi^{0} F_{\ell}\right]\right\} .
\end{aligned}
$$

Inserting the ansatz in the growth equations (24) yields

$$
\Gamma F_{\ell}=a^{3} c_{0} R_{\ell}^{*}, \quad \Gamma F_{u}=-a^{3} c_{0} R_{u}^{*} .
$$

\section{ANALYTICAL RESULTS}

For large wave numbers, $k \gtrsim 1$, we can neglect $f_{\ell}$ and the left-hand side in Eq. (26a). Analytical expressions for the growth rate can then be obtained in the limit of overlimiting and underlimiting current, respectively. In Appendices A and $\mathrm{B}$ we find that the growth rate can be expressed as

$$
\Gamma=a^{3} c_{0} k J^{0} \frac{\xi-\bar{\gamma} k^{2}}{\xi+k} .
$$


Here, in the linear underlimiting regime, $\xi$ is given as

$$
\begin{aligned}
\xi & =\left(1+\alpha_{\mathrm{a}}+\alpha_{\mathrm{c}}\right) K_{0} e^{\alpha_{\mathrm{c}} Z\left(\phi^{0}+V_{u}\right)}-\alpha_{\mathrm{a}} \frac{J^{0}}{c_{+}^{0}}, \\
c_{+}^{0} & =1-J^{0}, \quad \text { linear case, }
\end{aligned}
$$

while in the nonlinear overlimiting regime $\xi$ is

$$
\begin{aligned}
\xi & =2\left(\alpha_{\mathrm{a}}+\alpha_{\mathrm{c}}\right) K_{0} e^{\alpha_{\mathrm{c}} Z\left(\phi^{0}+V_{u}\right)}-\alpha_{\mathrm{a}} \frac{2 J^{0}}{c_{+}^{0}}, \\
c_{+}^{0} & \approx \frac{\bar{\lambda}_{\mathrm{D}}}{Z} \sqrt{\frac{2 J^{0}}{1-\frac{1}{J^{0}}}}, \quad \text { nonlinear case. }
\end{aligned}
$$

The factors $K_{0} e^{\alpha_{\mathrm{c}} Z\left(\phi^{0}+V_{u}\right)}$ are found by solving the zeroth-order reaction expression,

$$
J^{0}=R_{u}^{0}=K_{0}\left[c_{+}^{0} e^{\alpha_{\mathrm{c}} Z\left(\phi^{0}+V_{u}\right)}-e^{-\alpha_{\mathrm{a}} Z\left(\phi^{0}+V_{u}\right)}\right] .
$$

The charge-transfer coefficients $\alpha_{\mathrm{c}}$ and $\alpha_{\mathrm{a}}$ may depend on $\phi^{0}$ and $c_{+}^{0}$, so there is no general solution to Eq. (33). In the limit $K_{0} \ll 1$ the deposition term in Eq. (33) dominates, and we simply have

$$
K_{0} e^{\alpha_{\mathrm{c}} Z\left(\phi^{0}+V_{u}\right)}=\frac{J^{0}}{c_{+}^{0}} .
$$

In that limit $\xi$ becomes

$$
\begin{aligned}
& \xi=\left(1+\alpha_{\mathrm{c}}\right) \frac{J^{0}}{c_{+}^{0}}, \quad \text { linear case } \\
& \xi=2 \alpha_{\mathrm{c}} \frac{J^{0}}{c_{+}^{0}}, \quad \text { nonlinear case. }
\end{aligned}
$$

In the case of simple Butler-Volmer kinetics with constant charge transfer coefficients $\alpha_{\mathrm{c}}=\alpha_{\mathrm{a}}=\frac{1}{2}$, it is also possible to obtain simple solutions to Eq. (33). In that case we find

$$
\begin{aligned}
& \xi=\frac{J^{0}}{c_{+}^{0}}\left[\frac{1}{2}+\sqrt{1+4\left(\frac{K_{0}}{J^{0}}\right)^{2} c_{+}^{0}}\right], \text { linear, } \\
& \xi=\frac{J^{0}}{c_{+}^{0}} \sqrt{1+4\left(\frac{K_{0}}{J^{0}}\right)^{2} c_{+}^{0}}, \quad \text { nonlinear. }
\end{aligned}
$$

The critical wave number $k_{\mathrm{c}}$, where the perturbation is marginally stable, is found to be

$$
k_{\mathrm{c}}=\sqrt{\frac{\xi}{\bar{\gamma}}},
$$

and the wave number $k_{\max }$, at which the growth rate is maximum, is given as

$$
\begin{aligned}
k_{\max }= & \frac{\xi}{2}\left[\left(\frac{2-\xi \bar{\gamma}+2 \sqrt{1-\xi \bar{\gamma}}}{\xi \bar{\gamma}}\right)^{1 / 3}\right. \\
& \left.+\left(\frac{2-\xi \bar{\gamma}+2 \sqrt{1-\xi \bar{\gamma}}}{\xi \bar{\gamma}}\right)^{-1 / 3}-1\right] .
\end{aligned}
$$

We note that the analytical model takes the zeroth-order current density $J^{0}$ as input variable through $\xi$. If one wants the results as a function of the potential drop instead, a model of the system's current-voltage characteristic is needed. For simplicity, we just use the numerically calculated currentvoltage characteristic in the following.

To compute the results without reference to a numerical solution, an analytical model for the system's current-voltage characteristic is required. Such a model can be found in our previous work [16]. To obtain the total voltage drop over the system, the interfacial voltages from Eq. (18b) should also be taken into account.

\section{NUMERICAL SOLUTION}

The numerical simulations are carried out in the commercially available finite element software COMSOL MULTIPHYSICS ver. 4.3a. Following our previous work [16,23,26], the zerothand first-order problems are rewritten in weak form and implemented in the mathematics module of COMSOL. In the first-order problem we set the parameter $F_{u}$ to unity, meaning that the magnitude of the remaining first-order fields are given relative to the amplitude of the upper electrode perturbation. We choose to model the reaction using simple Butler-Volmer kinetics with $\alpha_{\mathrm{c}}=\alpha_{\mathrm{a}}=\frac{1}{2}$. To limit the parameter space, we choose fixed, physically reasonable values for the parameters listed in Table I. The values are chosen to correspond to copper electrodes in a copper sulfate solution. We note that the surface tension is quite difficult to determine experimentally, and most measurements are carried out at temperatures around $1000^{\circ} \mathrm{C}$ [27,28]. Ab initio calculations can give some impression of the behavior at lower temperatures [29], but these can hardly stand alone. Extrapolating the linear fit of Ref. [27] down to $0 \mathrm{~K}$ yields surface tension values close to those obtained from $a b$ initio calculations in Ref. [29]. This makes it somewhat plausible to apply the model from Ref. [27] in the region of interest around $300 \mathrm{~K}$. This yields a copper-gas surface energy of $1.92 \mathrm{~J} / \mathrm{m}^{2}$. The contact angle at the copper-water interface is very small [30], so finding the copper-water surface energy is just a matter of subtracting the surface energy of water from that of copper. The resulting surface energy is $\gamma \approx 1.85 \mathrm{~J} / \mathrm{m}^{2}$, as listed in Table I.

These choices leave us with three free parameters, which are the bias voltage $V_{0}$, the electrolyte concentration $c_{0}$, and the system length $L$.

TABLE I. Fixed parameter values used in the numerics.

\begin{tabular}{lcc}
\hline \hline Parameter & Symbol & Value \\
\hline Cation diffusivity [24] & $D_{+}$ & $0.714 \times 10^{-9} \mathrm{~m}^{2} \mathrm{~s}^{-1}$ \\
Anion diffusivity [24] & $D_{-}$ & $1.065 \times 10^{-9} \mathrm{~m}^{2} \mathrm{~s}^{-1}$ \\
Ion valence & $Z$ & 2 \\
Surface energy & $\gamma$ & $1.85 \mathrm{~J} \mathrm{~m}^{-2}$ \\
Temperature & $T$ & $300 \mathrm{~K}$ \\
Permittivity of water & $\epsilon_{\mathrm{w}}$ & $6.90 \times 10^{-10} \mathrm{~F} \mathrm{~m}^{-1}$ \\
Charge-transfer coefficients & $\alpha_{\mathrm{c}}, \alpha_{\mathrm{a}}$ & $\frac{1}{2}$ \\
Reaction constant $^{\mathrm{a}}$ & $k_{0}$ & $9.4 \times 10^{19} \mathrm{~m}^{-2} \mathrm{~s}^{-1}$ \\
Diameter of a copper atom $^{\mathrm{b}}$ & $a$ & $0.228 \mathrm{~nm}^{-}$ \\
\hline \hline
\end{tabular}

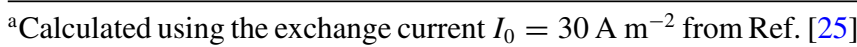
and $k_{0}=I_{0} /(Z e)$.

${ }^{\mathrm{b}}$ The cube root of the volume per atom in solid copper [24]. 


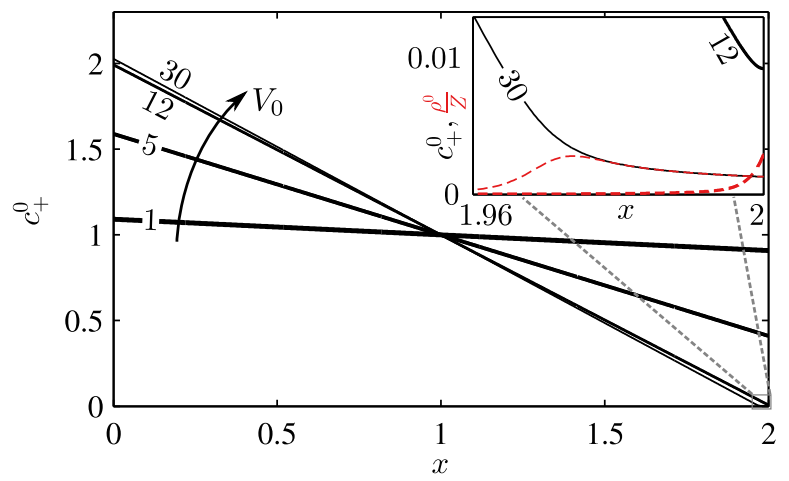

FIG. 2. (Color online) Zeroth-order cation concentrations $c_{+}^{0}$ shown in full (black) lines and zeroth-order charge densities $\rho^{0} / Z$ shown in dashed (red) lines. The inset shows the fields close to the electrode. In the simulation the parameter values $c_{0}=10 \mathrm{mM}$, $L=10 \mu \mathrm{m}$, and $V_{0}=\{1,5,12,30\}$ were used.

The solution procedure is as follows: First, the zeroth-order problem is solved for a given set of parameters. Then the first-order problem is solved for a range of wave numbers $k$. For each $k$ value, the corresponding growth rate $\Gamma$ and perturbation amplitude of the lower electrode, $F_{\ell}$, are obtained.

In Fig. 2 the zeroth-order cation concentrations $c_{+}^{0}$ and space-charge density $\rho^{0}$ are shown for $c_{0}=10 \mathrm{mM}, L=$ $10 \mu \mathrm{m}$, and varying bias voltage $V_{0}$. It is seen, that when the bias voltage exceeds $V_{0} \simeq 12$, local electroneutrality is violated near the cathode. For $V_{0}=30$ the nonequilibrium space-charge region extends far $(0.04 L)$ into the electrolyte.

\section{A. Results}

For plotting purposes we introduce the dimensionfull perturbation wavelength $\lambda=2 \pi L / k$. In Fig. 3 the growth rate $\Gamma$ is plotted versus $\lambda$ for $V_{0}=30, c_{0}=10 \mathrm{mM}$, and

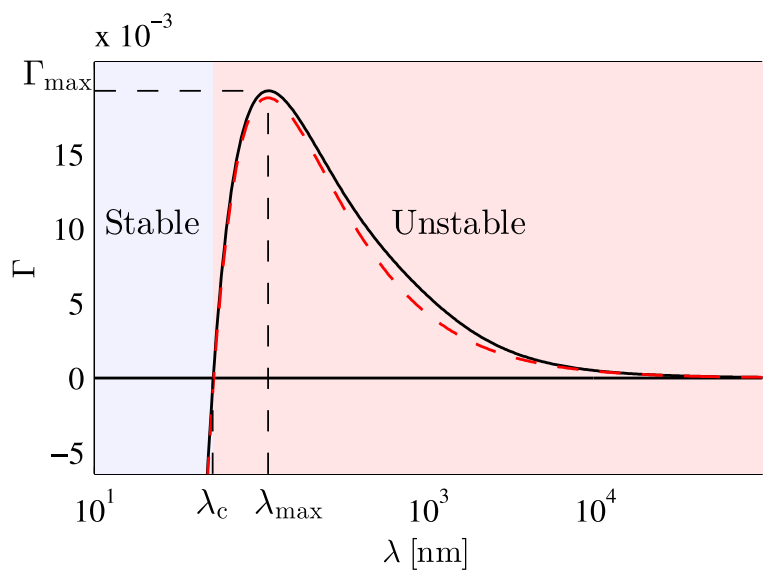

FIG. 3. (Color online) The growth rate $\Gamma$ plotted versus the perturbation wavelength $\lambda$ for $V_{0}=30, c_{0}=10 \mathrm{mM}$, and $L=10 \mu \mathrm{m}$. The full (black) line shows the growth rate obtained from numerical simulations, and the dashed (red) line shows the growth rate according to the analytical model [Eq. (29)]. For perturbation wavelengths smaller than the critical wavelength $\lambda_{\mathrm{c}}=51 \mathrm{~nm}$ the system is stable and for larger wavelengths it is unstable. At the most unstable wavelength $\lambda_{\max }=110 \mathrm{~nm}$ the growth rate is $\Gamma_{\max }=0.0193$.

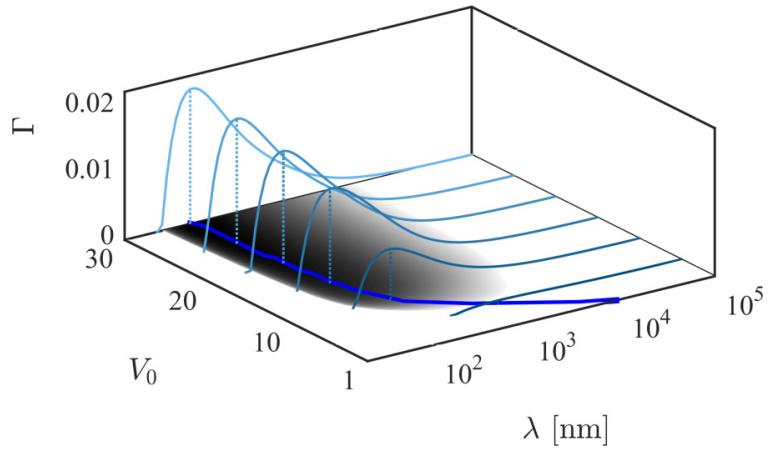

FIG. 4. (Color online) The growth rate $\Gamma$ plotted versus the perturbation wavelength $\lambda$ and voltage $V_{0}$ for $c_{0}=10 \mathrm{mM}$, and $L=10 \mu \mathrm{m}$. The (cyan) space curves are plots of $\Gamma$ versus $\lambda$ for $V_{0}=\{5,10,15,20,25,30\}$. The shade of the in plane contour plot is based on the logarithm of $\Gamma$, which is why there are no contours in the low $\lambda$ limit where $\Gamma$ is negative. The thick (blue) in plane line marks the crest of the hill; i.e., it marks the most unstable wavelength for each value of $V_{0}$.

$L=10 \mu \mathrm{m}$. Visible in the figure is a stable region for wavelengths smaller than the critical wavelength $\lambda_{\mathrm{c}}=51 \mathrm{~nm}$, and an unstable region for larger wavelengths. The most unstable wavelength we denote $\lambda_{\max }$, and the corresponding growth rate we denote $\Gamma_{\max }$.

To enable a more compact representation of the data, we introduce a gray-scale contour plot of the magnitude of $\Gamma$, as illustrated in Fig. 4. Here $\Gamma$ is plotted versus the wavelength $\lambda$ for $V_{0}=\{5,10,15,20,25,30\}$. The gray scale in the $\lambda-V_{0}$ plane is created by projecting the $\Gamma$ values from the above curves onto the plane. The solid (blue) line in the $\left(\lambda, V_{0}\right)$-plane marks the crest of the hill, thus representing the most unstable wavelength for each value of $V_{0}$.

In Fig. 5 we make use of the contour plots to show results for 12 sets of $\left(c_{0}, L\right)$-values. In each contour plot, $\Gamma$ is normalized by its maximum value, which is given above each plot. Shown in thick lines are $\lambda_{\max }$ in bright (yellow) and $\lambda_{\mathrm{c}}$ in black. The corresponding analytical results are shown in dashed (blue) and dotted (green) lines, respectively. The thin black lines show contours, where $\Gamma$ equals $\{0.01,0.2,0.7\}$ times the maximum value. There is a clear tendency in all of the panels that the growth rate $\Gamma$ increases rapidly with $V_{0}$, and the most unstable wavelength decreases as $V_{0}$ increases. Across the panels, the maximum growth rate is seen to increase for increasing $c_{0}$ and increasing $L$. Also, the most unstable wavelength $\lambda_{\max }$ and the critical wavelength $\lambda_{\mathrm{c}}$ become smaller as $c_{0}$ increases and as $L$ decreases.

A common feature seen in all of the panels is the kink in the $V_{0}$-versus- $\lambda_{\max }$ and $V_{0}$-versus- $\lambda_{\mathrm{c}}$ lines. At this kink, the slope of the lines changes markedly. The kink is located at the voltage, where the current reaches the limiting current, and it thus signifies that there is a qualitatively different behavior for over- and underlimiting current. This qualitative difference between the two regimes is in accordance with the analytical models. We also see that the kink voltage changes with $c_{0}$ and $L$. Specifically, it increases with $c_{0}$ and decreases with $L$. The main reason for this behavior is easily understood with reference to the zeroth-order Butler-Volmer reaction 


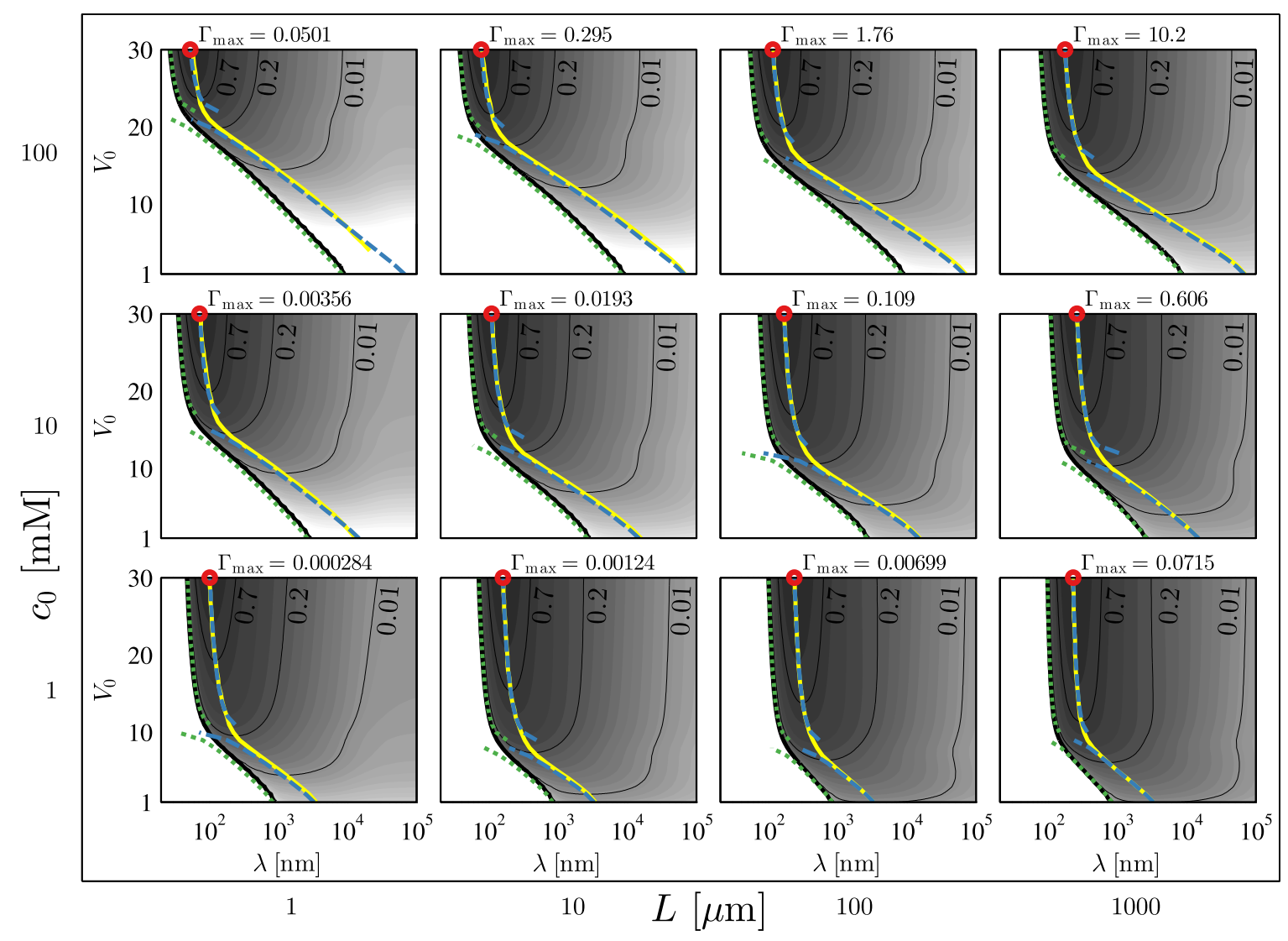

FIG. 5. (Color online) Contour plots of $\Gamma$ plotted versus wavelength $\lambda$ and voltage $V_{0}$ for $c_{0}=\{1 \mathrm{mM}, 10 \mathrm{mM}, 100 \mathrm{mM}\}$ and $L=$ $\{1 \mu \mathrm{m}, 10 \mu \mathrm{m}, 100 \mu \mathrm{m}, 1 \mathrm{~mm}\}$. In each plot, $\Gamma$ is normalized by its maximum value, and the contours are logarithmically spaced. The maximum value $\Gamma_{\max }$ of $\Gamma$ is given on top of each plot, and the point where the maximum value is attained is indicated with a dark (red) circle. The three thin black lines in each plot indicate contours where $\Gamma$ equals $0.01,0.2$, and 0.7 times $\Gamma_{\max }$. The thick bright (yellow) line marks $\lambda_{\max }$ for each value of $V_{0}$, and the dashed (blue) lines mark the two corresponding analytical limits. The thick black line marks $\lambda_{\mathrm{c}}$ for each value of $V_{0}$, and the dotted (green) lines mark the two corresponding analytical limits.

expression (18b). Setting the current in the system to the limiting current $J_{+}^{0}=1$, the reaction rates at the electrodes become

$$
\boldsymbol{e}_{x} \cdot \boldsymbol{n}_{p}=-K_{0}\left[c_{+} e^{\alpha_{\mathrm{c}} Z\left(\phi+V_{p}\right)}-e^{-\alpha_{\mathrm{a}} Z\left(\phi+V_{p}\right)}\right] .
$$

At the cathode, the first term in the bracket dominates, and at the anode the other. Therefore, both potential drops over the electrode interfaces scale as

$$
\Delta V \sim-\ln \left(K_{0}\right)=\ln \left(\frac{2 D_{+} c_{0}}{L}\right)-\ln \left(k_{0}\right),
$$

which increases monotonically with increasing $c_{0} / L$. As a consequence, the total potential drop at the limiting current also increases with increasing $c_{0} / L$, just as observed in Fig. 5.

In addition to the instability growth rate $\Gamma$, which gives a time scale for the development of instabilities, it is useful to have a measure for the characteristic instability length scale. For instance, we would like to estimate the thickness of the deposited layer, when instabilities start to develop. We define this instability length scale as the product of the zeroth-order growth rate Eq. (21) and the instability time scale at the most unstable wavelength

$$
L_{\Gamma}=L \frac{a^{3} c_{0} J_{+}^{0}}{\Gamma_{\max }},
$$

where the prefactor $L$ ensures a dimensionfull expression. In Fig. 6 we plot the instability length $L_{\Gamma}$ versus applied voltage $V_{0}$ for $L=100 \mu \mathrm{m}$ and varying $c_{0}$. The most unstable wavelength $\lambda_{\max }$ is also plotted in the same figure (dashed lines). It is seen that $L_{\Gamma}$ decreases as $V_{0}$ increases, but for small voltages $L_{\Gamma}$ is largest for high concentrations, while the

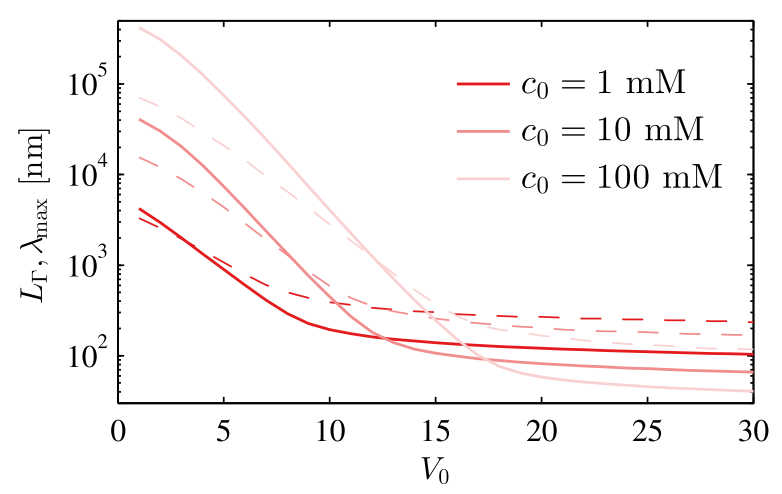

FIG. 6. (Color online) The instability length scale $L_{\Gamma}$ (full line) and most unstable wavelength $\lambda_{\max }$ (dashed line) plotted versus bias voltage $V_{0}$. The concentration varies between the values $c_{0}=$ $\{1 \mathrm{mM}, 10 \mathrm{mM}, 100 \mathrm{mM}\}$ and the length $L=100 \mu \mathrm{m}$ was used. 
opposite is true for high voltages. The reason for this reversal is that the interfacial voltage drops are largest for large $c_{0}$. At small voltages the bulk driving force in the systems with large $c_{0}$ is therefore small, and this causes the system to be less unstable than the low $c_{0}$ systems. We also see that $\lambda_{\max }$ scales in the same way as $L_{\Gamma}$. While the reason for this is not immediately obvious, it is seen to follow from the analytical expressions. Inserting Eq. (29) in Eq. (42) yields

$$
L_{\Gamma}=\frac{\lambda \max }{2 \pi} \frac{\xi+\frac{2 \pi L}{\lambda_{\max }}}{\xi-\bar{\gamma}\left(\frac{2 \pi L}{\lambda_{\max }}\right)^{2}},
$$

which confirms the approximate scaling between $L_{\Gamma}$ and $\lambda_{\max }$. The connection between $L_{\Gamma}$ and $\lambda_{\max }$ implies that $\lambda_{\max }$ sets the scale, not only for the variations in the horizontal direction, but also for variations in the vertical direction. We might therefore expect that the ramified electrodeposits, emerging at much longer times than $\Gamma_{\max }^{-1}$, have a universal length scale roughly set by $\lambda_{\max }$.

\section{DISCUSSION}

The main feature which sets our work apart from previous stability analyses of electrodeposition is the inclusion of the overlimiting regime. Presumably this regime has so far been avoided due to the nonlinearities arising at overlimiting current, which necessitate a more complicated treatment. However, the overlimiting regime is highly relevant for ramified growth problems $[7,8]$. As seen in Fig. 5, the instability growth rate increases markedly in the overlimiting regime, and there is also a change in qualitative behavior between the two regimes. Of course, the conclusions we reach, based on our model, are only strictly valid for planar electrodes. It does, however, seem reasonable to expect that the most unstable wavelength $\lambda_{\max }$ is comparable to the characteristic dimensions encountered in a ramified growth experiment. Our analysis can thus be used to rationalize experimental results. In this regard, our analytical models are particularly useful, since they allow for easy computation of the key quantities for other systems than the one treated here.

Perhaps the most important application of the stability analysis is as a means of validating more elaborate numerical models of ramified growth. A model of ramified growth must necessarily deal with a moving interface, and this, as well as other complications, makes for highly complex numerical models. To validate such models it is very useful to have a comparatively simple model, like the present one, to benchmark against in the relevant limit. Indeed, this was what originally motivated us to treat the stability problem.

An obvious shortcoming of the given analysis is the restriction to a steady-state zeroth-order solution. The principal reason for this choice is that it makes for a simpler problem. Furthermore, the numerical ramified growth model, to which we wish to compare our model, is at present also restricted to quasisteady state. In time, we wish to extend both models to the fully transient regime.

There is, however, some physical justification for making the steady-state assumption. As seen in Fig. 5, the growth rate $\Gamma$ is considerably smaller than unity in a large part of the investigated parameter space. The time it takes the system to reach steady state is given by the diffusive time, which in our normalization has the value one. Thus, as long as $\Gamma$ is much smaller than unity, the system reaches steady state long before any instabilities build up. In this case it is therefore justified to assume steady state. It should be noted that in this argument we make the reasonable assumption that the true growth rate in the transient regime does not significantly exceed the steady-state value.

In the numerical model we employ the widely used ButlerVolmer model with equal cathodic and anodic charge-transfer coefficients. The analytical model is however not restricted to this particular reaction model. In deriving the analytical results we only required that the charge-transfer coefficients vary slowly with the interfacial potential drop and the cation concentration. Thus, we expect the analytical model to apply equally well to Marcus kinetics and Butler-Volmer kinetics with asymmetric charge-transfer coefficients. Also, the effects of an electric double layer can be included implicitly by applying the Frumkin correction.

In future work, it would of course be interesting to extend the implicit general modeling of the electric double layers to explicit and more specific schemes, such as those presented in Refs. [31-34]. Also, it would be relevant to study the influence of advection on the morphological stability, in particular the effect of electroosmotic instabilities [17-19].

Finally, we hope that our results may inspire experimentalists in the field to analyze electrodeposition experiments in terms of our theoretical framework.

\section{CONCLUSION}

We have successfully solved the stability problem in the under- and overlimiting regime for the case of a copper sulfate solution trapped between two copper electrodes. In addition to the numerical solution of this particular problem, we have derived analytical solutions valid in either the overlimiting or the underlimiting limit. The behavior in the overlimiting regime differs qualitatively from the behavior in the underlimiting regime, and we find that the electrode becomes increasingly unstable as the current is increased above the limiting current. The stability analysis, and in particular the analytical limits, are valuable both for rationalizing experimental results and for validating more elaborate numerical models of ramified growth.

\section{APPENDIX A: THE ELECTRONEUTRAL LIMIT}

In the limit where the electrolyte is locally electroneutral and the time derivatives in the first-order transport problem are negligible, analytical solutions to the problem can be obtained. Setting the point of zero electrostatic potential at $x=1$, it is easily found that

$$
c=c_{+}=c_{-}=e^{Z \phi} \text {. }
$$

It follows that $c=e^{Z\left(\phi^{0}+\phi^{1}\right)} \approx e^{Z \phi^{0}}+e^{Z \phi^{0}} Z \phi^{1}$, and thus

$$
c^{0}=e^{Z \phi^{0}} \quad \text { and } \quad c^{1}=e^{Z \phi^{0}} Z \phi^{1} .
$$

Solving the zeroth-order problem yields

$$
c^{0}=1-J^{0}(x-1), \quad Z \phi^{0}=\ln \left[1-J^{0}(x-1)\right] .
$$


Using the electroneutrality assumption in Eq. (26a) we find

$$
0=\partial_{x}^{2} c^{*}-k^{2} c^{*}
$$

This equation has two solutions, but as long as the perturbation wavelength is considerably smaller than the electrode spacing, the solution which increases with $x$ is dominant:

$$
c^{*} \approx C e^{k(x-2)}
$$

where $C$ is a constant to be determined. From Eq. (A2) we then find

$$
\phi^{*}=\frac{1}{Z} \frac{c^{*}}{c^{0}}=\frac{C}{Z} \frac{e^{k(x-2)}}{1-J^{0}(x-1)} .
$$

At the upper electrode, $x=2$, the first-order reaction rate is (we set $F_{u}=1$ )

$$
\begin{aligned}
\frac{R_{u}^{*}}{K_{0}}= & e^{\alpha_{\mathrm{c}} Z\left(\phi^{0}+V_{u}\right)}\left[c^{*}+\partial_{x} c^{0}+c^{0}\left(\alpha_{\mathrm{a}}+\alpha_{\mathrm{c}}\right) Z\left(\phi^{*}+\partial_{x} \phi^{0}\right)\right] \\
& +\frac{R_{u}^{0}}{K_{0}}\left[\bar{\gamma} k^{2}-\alpha_{\mathrm{a}} Z\left(\phi^{*}+\partial_{x} \phi^{0}\right)\right] .
\end{aligned}
$$

Evaluating the fields at $x=2$, this expression becomes

$$
\begin{aligned}
\frac{R_{u}^{*}}{K_{0}}= & \left(1+\alpha_{\mathrm{a}}+\alpha_{\mathrm{c}}\right)\left(C-J^{0}\right) e^{\alpha_{\mathrm{c}} Z\left(\phi^{0}+V_{u}\right)} \\
& +\frac{J^{0}}{K_{0}}\left[\bar{\gamma} k^{2}-\alpha_{\mathrm{a}} \frac{C-J^{0}}{1-J^{0}}\right] .
\end{aligned}
$$

The first-order current into the upper electrode is $J^{*}=$ $-\partial_{x} c^{*}=-k C$, meaning that

$$
\begin{aligned}
-k C=R_{u}^{*}= & \left(1+\alpha_{\mathrm{a}}+\alpha_{\mathrm{c}}\right)\left(C-J^{0}\right) K_{0} e^{\alpha_{\mathrm{c}} Z\left(\phi^{0}+V_{u}\right)} \\
& +J^{0} \bar{\gamma} k^{2}-\alpha_{\mathrm{a}} J_{0} \frac{C-J^{0}}{1-J^{0}}
\end{aligned}
$$

and solving for $C$, we obtain

$$
C=J^{0} \frac{\left(1+\alpha_{\mathrm{a}}+\alpha_{\mathrm{c}}\right) K_{0} e^{\alpha_{\mathrm{c}} Z\left(\phi^{0}+V_{u}\right)}-\bar{\gamma} k^{2}-\alpha_{\mathrm{a}} \frac{J^{0}}{1-J^{0}}}{\left(1+\alpha_{\mathrm{a}}+\alpha_{\mathrm{c}}\right) K_{0} e^{\alpha_{\mathrm{c}} Z\left(\phi^{0}+V_{u}\right)}+k-\alpha_{\mathrm{a}} \frac{J^{0}}{1-J^{0}}} .
$$

The growth rate can be expressed as

$$
\Gamma=-a^{3} c_{0} J^{*}=a^{3} c_{0} k C
$$

so we have

$$
\Gamma=a^{3} c_{0} k J^{0} \frac{\xi-\bar{\gamma} k^{2}}{\xi+k},
$$

with the parameter $\xi$ given as

$$
\xi=\left(1+\alpha_{\mathrm{a}}+\alpha_{\mathrm{c}}\right) K_{0} e^{\alpha_{\mathrm{c}} Z\left(\phi^{0}+V_{u}\right)}-\alpha_{\mathrm{a}} \frac{J^{0}}{1-J^{0}} .
$$

To test whether the time derivatives in the first-order problem really are negligible, we compare the time derivative term $2 \Gamma c^{*}$ with the transverse diffusion term $k^{2} c^{*}$. Since Eq. (A12) implies $\Gamma \leqslant a^{3} c_{0} k J^{0}$, our assumption is justified if

$$
2 a^{3} c_{0} J^{0} \ll k
$$

Consequently, because $a^{3} c_{0} \ll 1$ for dilute systems and $J^{0}$ is of order unity, it is justified to neglect the time derivative, unless the perturbation wavelength is much larger than the electrode spacing.

The critical wave number $k_{c}$ is found by setting the nominator in Eq. (A12) equal to zero,

$$
k_{\mathrm{c}}=\sqrt{\frac{\xi}{\bar{\gamma}}} .
$$

To find the wave number $k_{\max }$, at which $\Gamma$ attains its maximum $\Gamma_{\max }$, we set the derivative of $\Gamma$ equal to zero and solve for $k$,

$$
\begin{aligned}
k_{\max }= & \frac{\xi}{2}\left[\left(\frac{2-\xi \bar{\gamma}+2 \sqrt{1-\xi \bar{\gamma}}}{\xi \bar{\gamma}}\right)^{1 / 3}\right. \\
& \left.+\left(\frac{2-\xi \bar{\gamma}+2 \sqrt{1-\xi \bar{\gamma}}}{\xi \bar{\gamma}}\right)^{-1 / 3}-1\right],
\end{aligned}
$$

with the asymptotic solutions,

$$
k_{\max } \approx \begin{cases}\left(\frac{\xi}{3 \bar{\gamma}}\right)^{1 / 2}, & \text { for } \quad \bar{\gamma} \xi \gg 1, \\ \left(\frac{\xi^{2}}{2 \bar{\gamma}}\right)^{1 / 3}-\frac{\xi}{2}, & \text { for } \quad \bar{\gamma} \xi \ll 1\end{cases}
$$

\section{APPENDIX B: THE STRONGLY NONLINEAR LIMIT}

In the limit where the driving force is very large, some of the terms in Eqs. (26a) and (26b) become dominant, which makes an analytical solution of the problem possible.

If the system is strongly driven, the field gradients are large close to the upper electrode, and this makes the electrode surface much more unstable. It follows that a larger $k$ value is needed for the surface tension to stabilize the system, so the most unstable value of $k$ will be larger than for less driven systems. In the strongly driven limit, we might therefore expect that Eq. (26b) largely is a balance between $\partial_{x}^{2} \phi^{*}$ and $k^{2} \phi^{*}$ in the region of interest. This leads us to making the ansatz

$$
\phi^{*}=\Phi e^{k(x-2)}
$$

where $\Phi$ is a constant. We now consider Eq. (26a) for the cation concentration, neglecting the left-hand side

$$
\begin{aligned}
0= & -\partial_{x}\left\{-\partial_{x} c_{+}^{*}-Z c_{+}^{*} \partial_{x} \phi^{0}-Z c_{+}^{0} \partial_{x} \phi^{*}\right\} \\
& -k^{2}\left(c_{+}^{*}+Z c_{+}^{0} \phi^{*}\right) .
\end{aligned}
$$

We assume that the terms $\partial_{x} c_{+}^{*}$ and $Z c_{+}^{*} \partial_{x} \phi^{0}$ are negligible compared to $Z c_{+}^{0} \partial_{x} \phi^{*}$ and insert the ansatz Eq. (B1),

$$
\begin{aligned}
0 & \approx Z \partial_{x} c_{+}^{0} k \phi^{*}+Z c_{+}^{0} k^{2} \phi^{*}-k^{2}\left(c_{+}^{*}+Z c_{+}^{0} \phi^{*}\right) \\
& \approx Z \partial_{x} c_{+}^{0} k \phi^{*}-k^{2} c_{+}^{*}
\end{aligned}
$$

implying that

$$
c_{+}^{*} \approx \frac{Z}{k} \partial_{x} c_{+}^{0} \phi^{*} .
$$

To test the assumptions leading to this result, we need expressions for $c_{+}^{0}, \partial_{x} c_{+}^{0}$, and $\partial_{x} \phi^{0}$. From Ref. [16] we have such expressions, and in the extended space-charge region (ESC) they take the simple forms

$$
c_{+}^{0}(x) \approx \sqrt{2} \frac{\bar{\lambda}}{Z} \sqrt{J^{0}}\left[x-1-\frac{1}{J^{0}}\right]^{-1 / 2},
$$




$$
\begin{aligned}
& \partial_{x} c_{+}^{0}(x) \approx-\frac{\sqrt{2}}{2} \frac{\bar{\lambda}}{Z} \sqrt{J^{0}}\left[x-1-\frac{1}{J^{0}}\right]^{-3 / 2}, \\
& \partial_{x} \phi^{0}(x) \approx-\frac{\sqrt{2}}{\bar{\lambda}_{\mathrm{D}}} \sqrt{J^{0}}\left[x-1-\frac{1}{J^{0}}\right]^{1 / 2} .
\end{aligned}
$$

The width of the ESC is given as $L_{\mathrm{ESC}}=1-1 / J^{0}$, so in the region close to the electrode, compared to the width of the ESC, the fields can be written as

$$
\begin{aligned}
c_{+}^{0}(x) & \approx \sqrt{2} \frac{\bar{\lambda}}{Z} \sqrt{J^{0}} L_{\mathrm{ESC}}^{-1 / 2}, \\
\partial_{x} c_{+}^{0}(x) & \approx-\frac{c_{+}^{0}}{2 L_{\mathrm{ESC}}}, \\
\partial_{x} \phi^{0}(x) & \approx-\frac{Z c_{+}^{0}}{\bar{\lambda}_{\mathrm{D}}^{2}} L_{\mathrm{ESC}} .
\end{aligned}
$$

Evaluating $\partial_{x} c_{+}^{*}$ we find

$$
\partial_{x} c_{+}^{*} \approx \frac{Z}{k} \frac{3 c_{+}^{0}}{4 L_{\mathrm{ESC}}^{2}} \phi^{*}-Z \frac{c_{+}^{0}}{2 L_{\mathrm{ESC}}} \phi^{*}
$$

which is seen to be much smaller than $Z c_{+}^{0} \partial_{x} \phi^{*}$ if

$$
2 k \gg \frac{1}{L_{\mathrm{ESC}}},
$$

that is, if the perturbation wavelength satisfies

$$
\bar{\lambda} \ll 4 \pi L_{\mathrm{ESC}}
$$

Similarly, we find that $Z c_{+}^{*} \partial_{x} \phi^{0}$ is much smaller than $Z c_{+}^{0} \partial_{x} \phi^{*}$ if

$$
\bar{\lambda}^{2} \ll \frac{8 \pi^{2}}{Z^{2}} \frac{\bar{\lambda}_{\mathrm{D}}^{2}}{c_{+}^{0}(2)} .
$$

Finally, the ansatz Eq. (B1) is justified if $2 \bar{\lambda}_{\mathrm{D}}^{2} k^{2} \phi^{*} \gg Z c_{+}^{*}$, which is equivalent to

$$
\bar{\lambda}^{3} \ll \frac{32 \pi^{3}}{Z^{2}} \frac{\bar{\lambda}_{\mathrm{D}}^{2}}{c_{+}^{0}(2)} L_{\mathrm{ESC}} .
$$

This last requirement is seen to follow if the two first requirements Eqs. (B14) and (B15) are fulfilled.

In the strongly driven regime, where Eqs. (B14) and (B15) are satisfied, the first-order current is approximately

$$
2 J_{+}^{*} \approx-Z c_{+}^{0} \partial_{x} \phi^{*}=-Z k c_{+}^{0} \Phi,
$$

at the upper electrode. The zeroth-order diffusive contribution is also very small at the upper electrode, meaning that we can simplify Eq. (A7)

$$
\begin{aligned}
R_{u}^{*} \approx & K_{0} e^{\alpha_{\mathrm{c}} Z\left(\phi^{0}+V_{u}\right)} c_{+}^{0}\left(\alpha_{\mathrm{a}}+\alpha_{\mathrm{c}}\right) Z\left(\phi^{*}+\partial_{x} \phi^{0}\right) \\
& +R_{u}^{0}\left[\bar{\gamma} k^{2}-\alpha_{\mathrm{a}} Z\left(\phi^{*}+\partial_{x} \phi^{0}\right)\right] \\
\approx & K_{0} e^{\alpha_{\mathrm{c}} Z\left(\phi^{0}+V_{u}\right)}\left(\alpha_{\mathrm{a}}+\alpha_{\mathrm{c}}\right)\left(c_{+}^{0} Z \Phi-2 J^{0}\right) \\
& +R_{u}^{0}\left[\bar{\gamma} k^{2}-\alpha_{\mathrm{a}}\left(Z \Phi-\frac{2 J^{0}}{c_{+}^{0}}\right)\right] .
\end{aligned}
$$

Inserting $R_{u}^{*}=J_{+}^{*} \approx-\frac{1}{2} Z k c_{+}^{0} \Phi$ we find

$$
\frac{Z}{2} k c_{+}^{0} \Phi=k J^{0} \frac{2\left(\alpha_{\mathrm{a}}+\alpha_{\mathrm{c}}\right) K_{0} e^{\alpha_{\mathrm{c}} Z\left(\phi^{0}+V_{u}\right)}-\bar{\gamma} k^{2}-\alpha_{\mathrm{a}} \frac{2 J^{0}}{c_{+}^{0}}}{2\left(\alpha_{\mathrm{a}}+\alpha_{\mathrm{c}}\right) K_{0} e^{\alpha_{\mathrm{c}} Z\left(\phi^{0}+V_{u}\right)}+k-\alpha_{\mathrm{a}} \frac{2 J^{0}}{c_{+}^{0}}},
$$

and since $\Gamma=-a^{3} c_{0} J_{+}^{*}$,

$$
\Gamma=a^{3} c_{0} k J^{0} \frac{\xi-\bar{\gamma} k^{2}}{\xi+k}
$$

with

$$
\xi=2\left(\alpha_{\mathrm{a}}+\alpha_{\mathrm{c}}\right) K_{0} e^{\alpha_{\mathrm{c}} Z\left(\phi^{0}+V_{u}\right)}-\alpha_{\mathrm{a}} \frac{2 J^{0}}{c_{+}^{0}} .
$$

Like in the electroneutral limit, neglecting the time derivative in the first-order problem is justified, unless the perturbation wavelength is much larger than the electrode spacing. The expressions (A15) and (A16) are also valid for the strongly nonlinear limit, if we use the nonlinear expression for $\xi$.
[1] G. Libbrecht, Rep. Prog. Phys. 68, 855 (2005).

[2] R. Trivedi and W. Kurz, Int. Mater. Rev. 39, 49 (1994).

[3] R. M. Brady and R. C. Ball, Nature (London) 309, 225 (1984).

[4] N. Nikolic, K. Popov, L. Pavlovic, and M. Pavlovic, Surf. Coat. Technol. 201, 560 (2006).

[5] G. Kahanda and M. Tomkiewicz, J. Electrochem. Soc. 136, 1497 (1989).

[6] C. Leger, J. Elezgaray, and F. Argoul, Phys. Rev. E 61, 5452 (2000).

[7] G. Gonzalez, M. Rosso, and E. Chassaing, Phys. Rev. E 78, 011601 (2008)

[8] J.-H. Han, E. Khoo, P. Bai, and M. Bazant, Sci. Rep. 4, 7056 (2014).
[9] P. Trigueros, J. Claret, F. Mas, and F. Sagues, J. Electroanal. Chem. 312, 219 (1991).

[10] O. Devos, C. Gabrielli, L. Beitone, C. Mace, E. Ostermann, and H. Perrot, J. Electroanal. Chem. 606, 75 (2007).

[11] K. Nishikawa, E. Chassaing, and M. Rosso, J. Electrochem. Soc. 160, D183 (2013).

[12] J.-N. Chazalviel, Phys. Rev. A 42, 7355 (1990).

[13] M. Rosso, Electrochim. Acta 53, 250 (2007).

[14] W. H. Smyrl and J. Newman, Trans. Faraday Soc. 63, 207 (1967).

[15] M. Rosso, J.-N. Chazalviel, and E. Chassaing, J. Electroanal. Chem. 587, 323 (2006).

[16] C. P. Nielsen and H. Bruus, Phys. Rev. E 89, 042405 (2014).

[17] I. Rubinstein and B. Zaltzman, Phys. Rev. E 62, 2238 (2000). 
[18] S. M. Rubinstein, G. Manukyan, A. Staicu, I. Rubinstein, B. Zaltzman, R. G. H. Lammertink, F. Mugele, and M. Wessling, Phys. Rev. Lett. 101, 236101 (2008).

[19] C. L. Druzgalski, M. B. Andersen, and A. Mani, Phys. Fluids 25, 110804 (2013).

[20] L. Sundstrom and F. Bark, Electrochim. Acta 40, 599 (1995).

[21] M. Z. Bazant, Acc. Chem. Res. 46, 1144 (2013).

[22] M. van Soestbergen, Russ. J. Electrochem. 48, 570 (2012).

[23] C. P. Nielsen and H. Bruus, Phys. Rev. E 90, 043020 (2014).

[24] D. R. Lide, CRC Handbook of Chemistry and Physics, 91st ed., edited by W. M. Haynes, (Internet Version 2011) (CRC Press/Taylor and Francis, Boca Raton, FL, 2010).

[25] D. R. Turner and G. R. Johnson, J. Electrochem. Soc. 109, 798 (1962).

[26] M. M. Gregersen, M. B. Andersen, G. Soni, C. Meinhart, and H. Bruus, Phys. Rev. E 79, 066316 (2009).
[27] H. Udin, A. J. Shaler, and J. Wulff, Trans. AIME 185, 186 (1949).

[28] V. K. Kumikov and K. B. Khokonov, J. Appl. Phys. 54, 1346 (1983).

[29] H. L. Skriver and N. M. Rosengaard, Phys. Rev. B 46, 7157 (1992).

[30] D. J. Trevoy and H. Johnson, J. Phys. Chem. 62, 833 (1958).

[31] L. H. Olesen, M. Z. Bazant, and H. Bruus, Phys. Rev. E 82, 011501 (2010).

[32] P. M. Biesheuvel, R. Zhao, S. Porada, and A. van der Wal, J. Colloid Interface Sci. 360, 239 (2011).

[33] T. R. Ferguson and M. Z. Bazant, J. Electrochem. Soc. 159, A1967 (2012).

[34] M. A. Quiroga, K.-H. Xue, T.-K. Nguyen, M. Tulodziecki, H. Huang, and A. A. Franco, J. Electrochem. Soc. 161, E3302 (2014). 